\title{
Downregulation of TRIM28 inhibits growth and increases apoptosis of nude mice with non-small cell lung cancer xenografts
}

\author{
LEI LIU $^{1}$, LEI ZHANG ${ }^{2}$, JIANPING WANG ${ }^{1}$, XUERONG ZHAO ${ }^{1}$, QIAN XU $^{3}$, YANJIE LU ${ }^{4}$, \\ YANZHEN ZUO ${ }^{4}$, LONG CHEN $^{3}$, JIA DU $^{5}$, YALI LIAN $^{5}$ and QIN ZHANG $^{5}$ \\ ${ }^{1}$ Department of Immunology, Basic Medical Institute; ${ }^{2}$ Department of Community Care, Nursing Institute; \\ ${ }^{3}$ Department of Central Laboratory; ${ }^{4}$ Department of Pathology, Basic Medical Institute; ${ }^{5}$ Grade 2013, \\ Clinical Institute, Chengde Medical College, Chengde, Hebei 067000, P.R. China
}

Received July 23, 2017; Accepted October 24, 2017

DOI: $10.3892 / \mathrm{mmr} .2017 .7955$

\begin{abstract}
TRIM28 is a well-known transcriptional co-repressor of Kruppel-associated box zinc finger proteins. The authors previously demonstrated that TRIM28 small interfering (si)RNA decreases cell proliferation and inhibits cell cycle progression in non-small cell lung cancer (NSCLC) cell lines. The present study further demonstrated that the stable silencing of TRIM28 expression by a specific siRNA lentivirus vector significantly inhibited the growth and exerted obvious anti-tumor effects in nude mice. The results of the terminal deoxynucleotidyl transferase-mediated deoxyuridine triphosphate nick-end labeling assay indicated that TRIM28 knockdown increased apoptosis. Furthermore, TRIM28 knockdown decreased the expression of $\mathrm{B}$ cell lymphoma (Bcl)-2 and increased the expression of $\mathrm{Bcl}-2$ associated $\mathrm{X}$, apoptosis regulator and $\mathrm{p} 53$ at the gene and protein levels. Auto-antibodies to TRIM28 were present in $12.32 \%$ of the sera of the patients with NSCLC. The results suggest that TRIM28 knockdown may be effective against NSCLC, and TRIM28 antibodies have the potential to act as novel diagnostic and therapeutic tools.
\end{abstract}

\section{Introduction}

The 5-year survival rate for early-stage non-small cell lung cancer (NSCLC) patients who are eligible for curative surgery (1) varies from approximately $66.3 \%$ (stage IB) to $83.9 \%$ (stage IA) (2), whereas, the 5-year survival rate of patients with unresectable NSCLC is 5 to $17 \%$ even after chemo-radiation therapy (3). Current techniques, such as computed tomography, magnetic resonance imaging, positron emission tomography, sputum cytology, and histological

Correspondence to: Dr Lei Liu, Department of Immunology, Basic Medical Institute, Chengde Medical College, Anyuan Road, Shuangqiao, Chengde, Hebei 067000, P.R. China

E-mail: homingreceptor@hotmail.com

Key words: non-small cell lung cancer, TRIM28, apoptosis, nude mice, ELISA, small interfering RNA, PAa examination of bronchoscopic tissues (4), are expensive or impractical for large-scale population screening of early-stage NSCLC. Therefore, the identification of efficient and accurate tools for early detection is crucial.

Our previous study demonstrated that the expression of the TRIM28 gene was high in NSCLC cell lines and tissues. TRIM28 provided a survival advantage to lung cancer cells, because TRIM28 knockdown decreased cell proliferation and inhibited cell cycle progression in NSCLC cell lines (5). TRIM28 is an important member of the tripartite motif superfamily which regulats embryonic development (6), chromatin organization, erythroblast differentiation (7), and the DNA damage response (8-11). Moreover, TRIM28 acts as a universal corepressor of KRAB zinc finger proteins. Studies have shown that TRIM28 also plays a role in the carcinogenesis and metastasis of cervical cancer (12), breast cancer (13), colorectal cancer (14), gastric cancer (15), kaposi's sarcoma (16), and ovarian cancer $(17,18)$. The downregulation of TRIM28 gene expression reduced the ability of cancer stem cells to self-renew, resulting in a significant reduction of tumor growth. The transforming growth factor- $\beta$ (TGF- $\beta$ )-induced epithelial-mesenchymal transition was impaired by shRNAi-mediated TRIM28 depletion via the acetylation and methylation of histones on E-cadherin and N-cadherin promoters (19). Loss of function of TRIM28 led to dysregulation of the cell cycle, cellular response to stress, cancer cell metabolism, and inhibition of oxidative phosphorylation (20). Despite these well-documented biological functions of TRIM28, little is known about the mechanisms underlying the role of TRIM28 in NSCLC.

Serum marker detection is a high-profile topic for auxiliary diagnosis of early NSCLC, and its advantages include easy operation, low price, noninvasiveness, accessibility of samples, and the ability for continuous monitoring (21). Clinical studies have examined various indicators. Halvorsen et al (22) validated six microRNAs (miR-429, miR-205, miR-200b, miR-203, miR-125b, and miR-34b) that were candidate biomarkers for future screening detection, because their abundance in the serum of NSCLC patients was significantly higher than that in chronic obstructive pulmonary diseases (COPD) patients and healthy volunteers. The serum laminin levels measured by solid-phase sandwich enzyme-linked immunosorbent assay 
(ELISA) were significantly higher in NSCLC patients, and this method might be promising as a diagnostic supplement (23). The level of four serum markers (CEA, CYFRA21-1, NSE and miR-21) was measured in 50 NSCLC patients and 60 healthy donors, and the results indicated that serum miR-21 had the highest diagnostic value, whereas the combination of miR-21 and CYFRA21-1 improved the diagnostic efficiency for early NSCLC (24). The serum of patients with lung cancer was analyzed by mass spectrometry, and a total of 17 distinct predictive proteins were identified in NSCLC patients with metastasis compared to healthy controls (25).

In this study, we observed that TRIM28 knockdown exerted anti-tumor and pro-apoptotic activity using a nude mouse xenograft tumor model of NSCLC. Here, using TRIM28 as the tumor-associated antigen, we also determined the suitability of reverse ELISA for the detection of specific antibodies in the serum of NSCLC patients to develop an early diagnostic method and an effective therapeutic strategy for NSCLC.

\section{Patients and methods}

Patients and samples. Serum samples from 138 NSCLC patients and 80 healthy controls were collected between November 2012 and December 2016 in the Affiliated Hospital of the Chengde Medical College Cancer Center (Chengde, China). The cases were confirmed by histopathological and clinical diagnosis. The cases involving autoimmune disease, systemic inflammatory conditions, or the use of immunosuppressive agents were excluded from the study. Tumor differentiation and clinical stages were classified according to the fifth edition of the tumor-node-metastasis classification of the International Union Against Cancer. Patient recruitment and serum analysis were conducted under written informed consent and the study was approved by the Research Ethics Review Committee of Chengde Medical College (Chengde, China).

Reverse ELISA. High-binding 96-well microtiter plates (Costar, Cambridge, MA, USA) were coated with $200 \mathrm{mg} / \mathrm{ml}$ of TRIM28 recombinant protein (Abcam, Cambridge, UK) diluted in $0.1 \mathrm{M} \mathrm{NaHCO} 3(\mathrm{pH} 8.3)$ overnight at $4^{\circ} \mathrm{C}$. The plates were washed three times with PBS containing Tween-20 and blocked with $1 \%$ bovine serum albumin (BSA) for $2 \mathrm{~h}$ at $37^{\circ} \mathrm{C}$. Serum samples were serially diluted in PBS containing $1 \% \mathrm{BSA}$, and $100 \mu \mathrm{l}$ of the diluted samples were applied to microwells and incubated overnight at $4^{\circ} \mathrm{C}$. Then, the plates were washed three times and incubated for $2 \mathrm{~h}$ at $37^{\circ} \mathrm{C}$ in goat anti-human HRP-conjugated secondary antibody (PharMingen, San Diego, CA, USA) diluted in PBS. After washing, the microwells were developed using TMB Peroxidase Substrate solution and the absorbance was read at $450 \mathrm{~nm}$ using a microplate autoreader.

The standard serum was assigned a value of $100 \mathrm{U} / \mathrm{ml} \mathrm{rabbit}$ anti-human TRIM28 PcAbs (Abcam). Positivity of TRIM28 autoantibodies for an individual patient was defined as an absorbance value greater than two standard deviations (SD) above the mean absorbance of non-cancer control sera.

Cell culture and RNA interference assay. The protocol was described in our previous study (26). The human NSCLC cell line PAa (BioVector NTCC Inc., Shanghai, China) was grown in a monolayer in Dulbecco's modified Eagle's medium (DMEM) supplemented with $10 \%$ fetal bovine serum (FCS) and was maintained at $37^{\circ} \mathrm{C}$ in an atmosphere of humidified air with $5 \% \mathrm{CO}_{2}$. Complementary oligonucleotides containing a short hairpin RNA (shRNA) targeting TRIM28 were dimerized and cloned into the pLVTHM lentiviral vector (Shanghai GeneChem Co., Ltd., Shanghai, China). The recombinant lentiviral vector and a lentiviral package plasmid were cotransfected into 293T cells to produce the lentiviral particles. PAa cells infected with lentiviral particles containing GFP were selected by flow cytometry sorting using an anti-GFP monoclonal antibody (Abcam) for culture and expansion. The stable transfected PAa cells were cultured for $48 \mathrm{~h}$, and the expression of TRIM28 at the mRNA and protein levels was examined by reverse transcription-polymerase chain reaction (RT-PCR) and western blot analysis.

Tumorigenicity in nude mice. All animal studies were approved by the Research Ethics Review Committee of Chengde Medical College (Chengde, China). Twenty female BALB/c nude mice (Vital River Laboratories Co., Ltd., Beijing, China) at 4 weeks of age were divided into two groups of 10 animals. The two groups of mice were subcutaneously injected with PAa/control-small interfering (si)RNA and PAa/TRIM28-siRNA cells, respectively. Mice were observed daily for viability and changes in general health and behavior. The body weight of the mice was monitored weekly. Tumor volume was assessed by measuring the length and width, and a standard equation was used for the calculation of tumor volume: Tumor volume $=$ length $\mathrm{x}$ width ${ }^{2} \mathrm{x} 0.52$, and was calculated for depicting a growth curve. Except for mice with large tumor burdens, animals were sacrificed 4 weeks after injection by cervical dislocation. Average tumor size was estimated by physical measurement of the excised tumor.

Terminal deoxynucleotidyl transferase-mediated deoxyuridine triphosphate nick-end labeling (TUNEL) assay. Apoptosis was quantitatively analyzed by using the in situ apoptosis detection TUNEL kit (Beyotime Institute of Biotechnology, Beijing, China) according to the manufacturer's instructions. Five fields were randomly chosen under a fluorescence microscope for each slide of each section (BZ-9000; Keyence Corporation, Osaka, Japan). The apoptosis index (AI) was calculated as the number of apoptotic cells/total cell number x100\%. Assays were performed in a blinded manner.

Immunohistochemistry. The tumors were collected from nude mice and processed into $5 \mu \mathrm{m}$ slices. Paraffin sections were stained with hematoxylin and eosin (H\&E; Beyotime Institute of Biotechnology). Mouse immunohistochemical studies for Ki-67 were conducted by using the streptavidin-peroxidase method (Zhongshan Golden Bridge Biotech Co., Ltd., Beijing, China). The tissue sections were deparaffinized with xylene and rehydrated with decreasing concentrations of ethanol. The sample slices were incubated overnight using a primary rabbit polyclonal antibody against Ki-67 (dilution of 1:100; ProteinTech Group, Inc., Chicago, IL, USA) in a humidified container at $4^{\circ} \mathrm{C}$. Immunohistochemistry was performed on a non-biotin horseradish-peroxidase detection system according to the manufacturer's instructions. Images were acquired using a Leica IX71 microscope. 
Reverse transcription-quantitative polymerase chain reaction $(R T-q P C R)$. Total RNA was extracted from tumor specimens using TRIzol reagent (Invitrogen, Carlsbad, CA, USA) according to the manufacturer's protocol. cDNA was synthesized from $2 \mu \mathrm{g}$ of total RNA using a reverse transcription kit (Takara, Dalian, China). PCRs included the following components: $100 \mathrm{nmol} / \mathrm{l}$ of each primer, diluted cDNA templates, and SYBR-Green PCR Master mix (Applied Biosystems, Warrington, UK), to a total volume of $20 \mu \mathrm{l}$. The real-time quantitative PCR assays were conducted with an ABI Prism 7000 SDS instrument (Applied Biosystems Life Technologies, Foster City, CA, USA). Reactions were incubated at $94^{\circ} \mathrm{C}$ for 4 min and then amplified using temperature cycles of $94^{\circ} \mathrm{C}$ for $15 \mathrm{sec}, 58^{\circ} \mathrm{C}$ for $30 \mathrm{sec}, 72^{\circ} \mathrm{C}$ for $35 \mathrm{sec}$. Amplifications were carried out for 45 cycles, followed by a $3 \mathrm{~min}$ extension at $72^{\circ} \mathrm{C}$. All reactions were performed in triplicate and the data were converted to fold change and normalized to the expression level of GAPDH, which served as an endogenous control. The relative gene expression levels were calculated using the following equation: 2 - [Ct B cell lymphoma $(\mathrm{Bcl})-2 / \mathrm{Bcl}-2$ associated $\mathrm{X}$ protein $(\mathrm{Bax})-\mathrm{Ct}$ control]. The primer sequences were as follows: Bcl-2 F,-5-TAC GAGTGGGATGCGGGAGATGT-3 and R,-5-CCACCGAAC TCAAAGAAGGC-3; Bax F,-5-CACCAGCTCTGAGCAGA TCA-3 and R,-5-ATGTCAGCTGCCACTCGGA-3; GAPDH F,-5-CAATGACCCCTTCATTGACC-3 and R,-5-TGGAAG ATGGTGATGGGATT-3; and p53 F,-5-GCAGTTCCTCTT CCTGCAGTACTC-3 and R, 5-AACCAGACCTCAGGCGGC TCATAG-3 (Shenggong Biotech Co., Ltd., Shanghai, China).

Western blot analysis. Tumor tissues were lysed in ice-cold lysis buffer containing $50 \mathrm{mmol} / \mathrm{l}$ Tris- $\mathrm{HCl}(\mathrm{pH} 8.0), 150 \mathrm{mmol} / \mathrm{l}$ $\mathrm{NaCl}, 0.5 \% \mathrm{NP} 40,0.5 \%$ sodium deoxycholate, $0.1 \%$ sodium dodecyl sulfate (SDS), plus protease inhibitor (Calbiochem, Darmstadt, Germany). Equal amounts of proteins were subjected to SDS-polyacrylamide gel electrophoresis using a $12 \%$ running gel. The gel-separated proteins were transferred to polyvinylidene difluoride membranes (Millipore, Bedford, MA, USA), and the membranes were blocked with $0.5 \%$ milk in PBS for $1 \mathrm{~h}$ at room temperature. The membranes were washed in Tris-buffered saline Tween (TBST), incubated with rabbit monoclonal Bcl-2, Bax or p53 antibody (dilution of 1:500; ProteinTech Group, Inc.) overnight at $4^{\circ} \mathrm{C}$, washed again in TBST, incubated with horseradish peroxidase-conjugated goat anti-rabbit $\mathrm{IgG}$ for $1 \mathrm{~h}$ at room temperature, washed again in TBST, and detected by chemiluminescence using the ECL detection kit (Amersham, Buckinghamshire, UK).

Statistical analysis. Data were presented as mean and SD. Analysis of variance followed by Bonferroni's test was used to determine the significant differences between groups. $\mathrm{P}<0.05$ was considered significant. All statistical analyses were conducted using software version SPSS 17.0 (SPSS, Inc., Chicago, IL, USA).

\section{Results}

Knockdown of TRIM28 inhibited the tumor growth in vivo. TRIM28 specific siRNA lentivirus vector and a negative control vector (si-control) were designed and constructed to silence TRIM28 in lung adenocarcinoma cell PAa. Nude mice received an injection of PAa/TRIM28-siRNA cells and PAa/control-siRNA cells for 4 weeks (5 times/week). Western blot assay were used to confirm TRIM28 siRNA led to a marked reduction of protein levels both in PAa cell line and tumor tissue from nude mice $(\mathrm{P}<0.001$, relative to the si-control; Fig. 1A and B).

The tumor growth of nude mice was assessed during the 4-week treatment period. TRIM28 siRNA inhibited tumor growth, and the inhibition was significantly higher than that of PAa/control-siRNA (Fig. 1C). Tumors were developed within 1 week in mice injected with PAa/control-siRNA. In the PAa/TRIM28-siRNA treated group, tumor formation was delayed by more than 1 week compared with the control group (Fig. 1D). Tumor size in mice from the PAa/TRIM28-siRNA group was significantly smaller than in the PAa/control-siRNA group ( $110 \pm 15$ vs. $294 \pm 27 \mathrm{~mm}^{3}$ at 3 weeks, $\mathrm{P}<0.001$, and $155 \pm 14$ vs. $407 \pm 24 \mathrm{~mm}^{3}$ at 4 weeks, $\mathrm{P}<0.001$ ) (Fig. 1D). Furthermore, the treatment with PAa/TRIM28-siRNA significantly decreased tumor weight $(0.71 \pm 0.28 \mathrm{~g})$ compared with PAa/control-siRNA $(1.67 \pm 0.81 \mathrm{~g}, \mathrm{P}<0.001$; Fig. 1E). None of the evaluated mice lost body weight during the experimental period (Fig. 1F).

TRIM28 siRNA stimulated apoptosis in a nude mouse xenograft tumor model. We used histology to evaluate the effect of TRIM28 siRNA on tumor growth in the nude mice. $H \& E$ staining of tumor tissues demonstrated that microvessels filled with erythrocytes were abundant in tumor tissues from mice injected with PAa/control-siRNA cells; however, a few microvessels were observed in tumors treated with PAa/TRIM28-siRNA (Fig. 2A, upper panel). Cell proliferation was detected by Ki-67 staining. The number of cells that stained positive for Ki-67 in tumor tissues from mice treated with PAa/TRIM28-siRNA $(4.13 \pm 1.42 \%)$ was significantly smaller than in mice injected with PAa/control-siRNA cells (14.23 $\pm 2.49 \%, P=0.013$; Fig. 2A, middle panel, and Fig. 2B). The degree of apoptosis was analyzed by conducting a TUNEL assay. The knockdown of TRIM28 significantly induced apoptosis compared to the control group (Fig. 2A, lower panel). The percentage of total nuclei in TUNEL-positive cells in mice injected with PAa/TRIM28-siRNA and PAa/control-siRNA was $21.67 \pm 2.58$ and $5.08 \pm 1.89 \%$, respectively $(\mathrm{P}<0.001$; Fig. 2A, lower panel, and Fig. 2C).

Effects of TRIM28 knockdown on the expression levels of $B c l-2$, Bax, and p53. Tumors were removed from mice after treatment, and the expression levels of the anti-apoptotic gene Bcl-2 and pro-apoptotic genes Bax and p53 were measured by real-time PCR and western-blotting. The expression of Bcl-2 was downregulated (Fig. 3A, D and E) whereas the expression of Bax (Fig. 3B, D and E) and p53 (Fig. 3C-E) was upregulated at both the mRNA and protein levels in the tumor tissues from mice injected the PAa/TRIM28-siRNA cells compared to the levels in the control-siRNA group $(\mathrm{P}<0.001)$. These results suggest that the knockdown of TRIM28 expression in nude mice models caused differential expression of pro- and anti-apoptotic genes.

Detection of TRIM28 serum autoantibodies in NSCLC patients. In our study, 138 NSCLC patients and 80 healthy donors were 

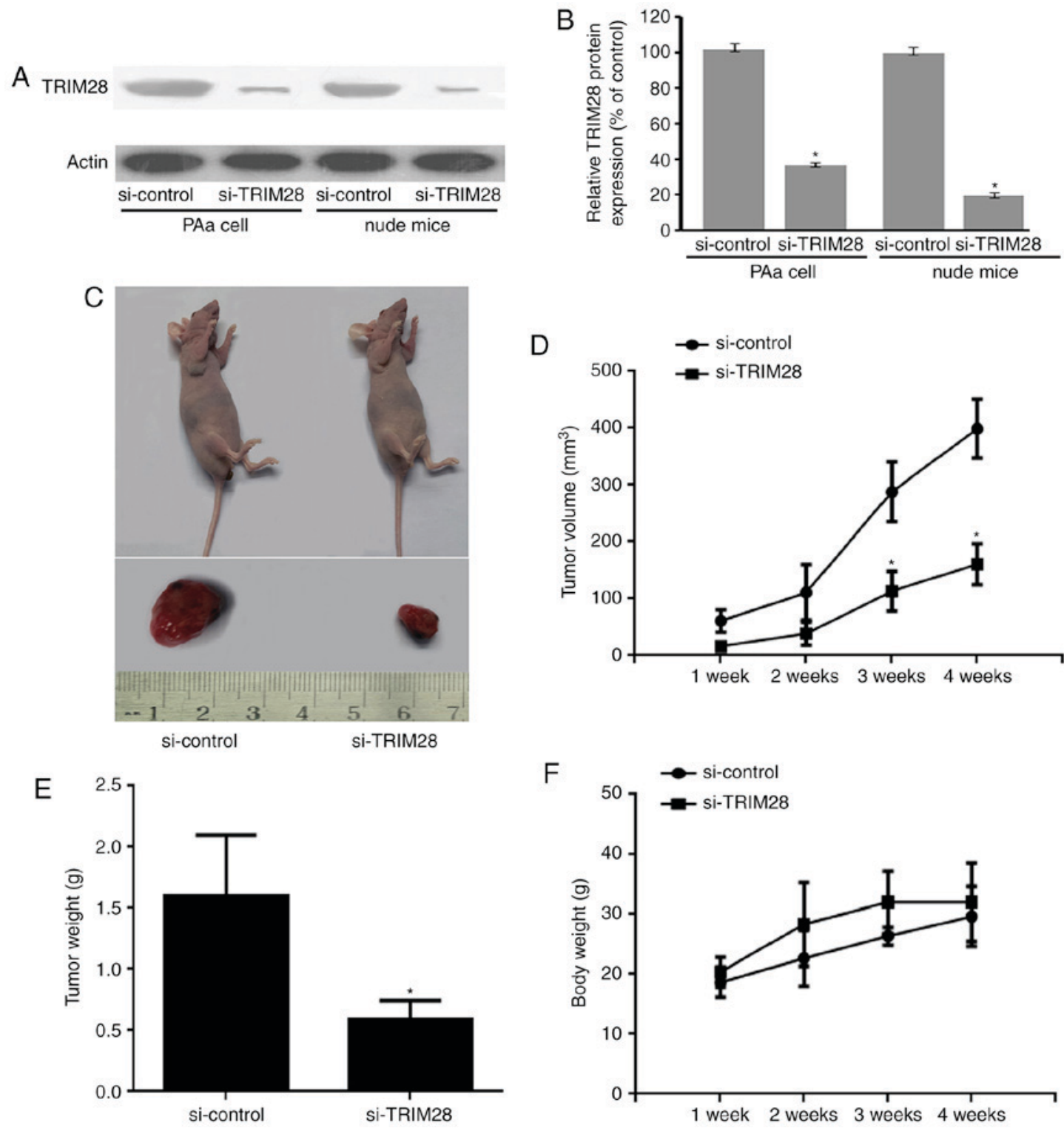

Figure 1. Tumor growth was inhibited by the knockdown of TRIM28 in nude mice. (A) Representative western blot analysis of TRIM28 and $\beta$-actin proteins from PAa cells transfected with siRNAs and tumors excised from two groups. (B) The expression level of each band was measured by densitometry and normalized to the corresponding $\beta$-actin. The data represent mean $\pm \mathrm{SD}(\mathrm{n}=3) ;{ }^{*} \mathrm{P}<0.001$ compared to the si-control group. (C) Top, images of representative mice with tumor. Bottom, images of tumors excised from two groups. (D) The tumor growth curve was significantly inhibited in the TRIM28-siRNA group. (E) Tumors were dissected after 4 weeks of treatment and the tumor weight was recorded. (F) No loss of body weight was observed in mice. ${ }^{*} \mathrm{P}<0.001$ compared to the si-control group. si, small interfering; SD, standard deviation.

recruited. Autoantibodies to TRIM28 were detected in the sera of 17 NSCLC patients but in none of the healthy controls $(\mathrm{P}=0.001)$, corresponding to a sensitivity of $12.32 \%(17 / 138)$ and a specificity of $100 \%$ (Fig. 4). Of note, antibodies to TRIM28 were detected in $14.43 \%$ (14/97) of patients with early stage I and II disease and $7.32 \%$ (3/41) of patients with more advanced disease (stage III and IV). The positive detection rate of TRIM28 was non-significantly higher in adenocarcinomas $(18.97 \%, 11 / 58)$ than in squamous epithelial carcinoma $(9.80 \%$, $4 / 51)$ and large cell lung cancer $(6.90 \%, 2 / 29)$ (Table I). The relationship between the detection of TRIM28 antibodies and the clinical outcome of NSCLC patients was investigated by conducting a 70-month follow-up of 97 early-stage patients. The incidence of recurrence and/or metastasis was significantly higher $(\mathrm{P}=0.001)$ in the patients with TRIM28 antibodies $(85.7 \%, 12$ of 14$)$ than in those without TRIM28 antibodies
(39.8\%, 33 of 83). Of note was that among these 12 patients with TRIM28 antibodies, 10 died during follow-up.

\section{Discussion}

TRIM28 is an important regulator of carcinogenesis in several neoplasms. Addison et al (13) showed that TRIM28 was overexpressed in breast tumors and the depletion of TRIM28 in breast cancer cell lines slowed cell proliferation and inhibited tumor growth and metastasis in mice xenografts models. Bojkowska et al (27) demonstrated that liver-specific TRIM28 knockout induced strikingly sexually dimorphic phenotypic disturbances, and developed male-specific steatosis and secondary hepatic tumors. The expression of the TRIM28 gene was significantly higher in gastric cancer tissues and the proliferation rate of cancer and resistance to anoikis were 
A
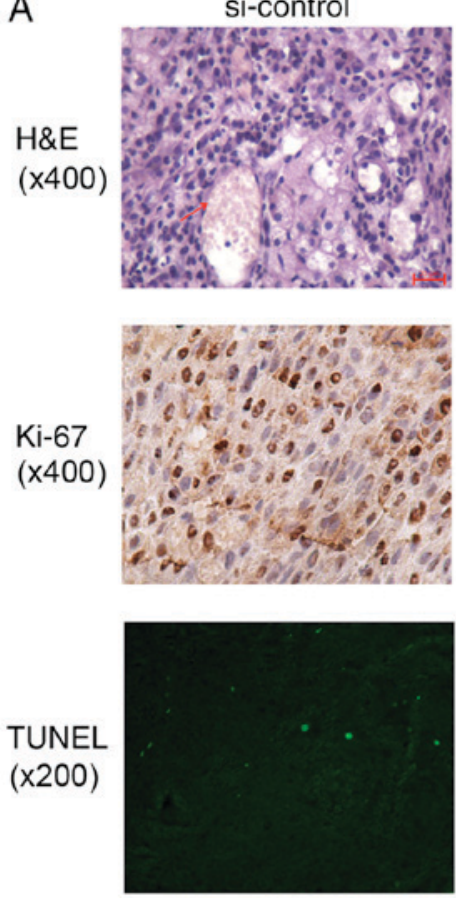

Si-TRIM28
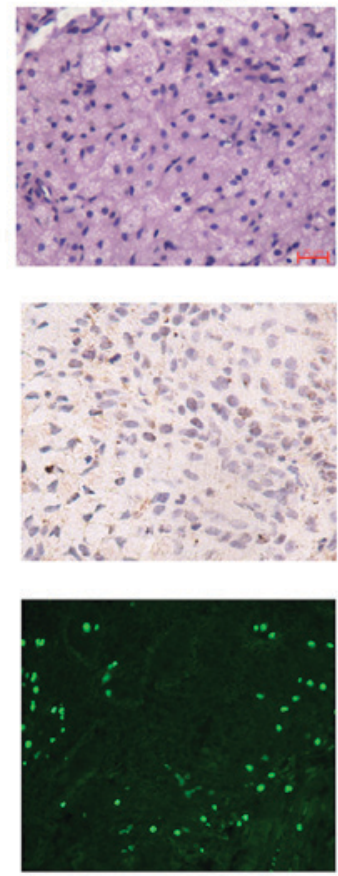

$\mathrm{B}$
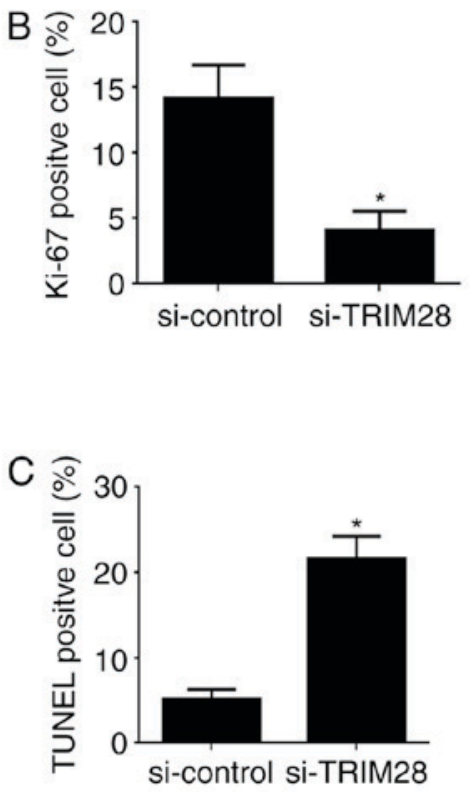

Figure 2. Histology and immunohistochemistry of tumor tissues from xenografted nude mice. (A) Top, H\&E staining. As indicated by the red arrow, the red-stained material in tumors from the control group is erythrocytes and the adjacent structures are blood vessels, which are rarely observed in tumors from PAa/TRIM28-siRNA treated mice (magnification, x400). Middle, immunostaining for Ki-67 (magnification, x400). Bottom, TUNEL staining (magnification, $\mathrm{x} 200$ ). (B) The percentages of Ki-67-positive nuclei was determined on four slides for each group and expressed as mean \pm SD. (C) Analysis of the degree of apoptosis in tumors from mice. " $\mathrm{P}<0.001$ compared to the si-control group. H\&E, hematoxylin and eosin; si, small interfering; TUNEL, terminal deoxynucleotidyl transferase-mediated deoxyuridine triphosphate nick-end labeling; SD, standard deviation.

A

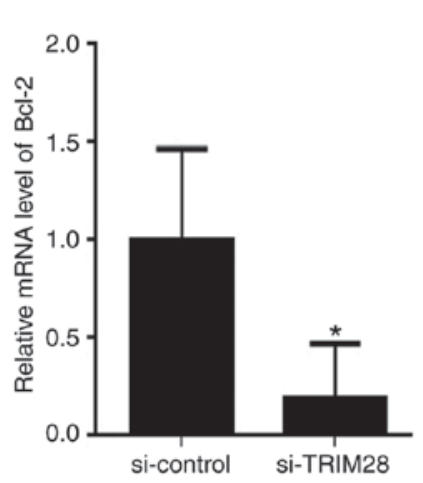

D

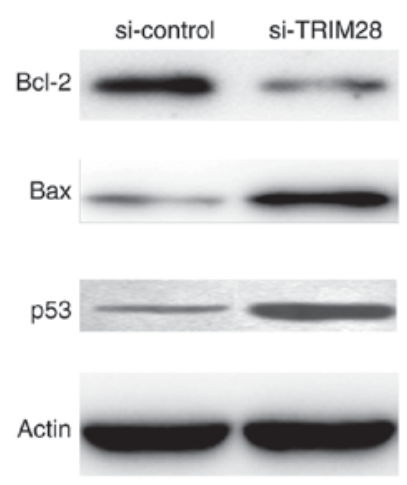

B

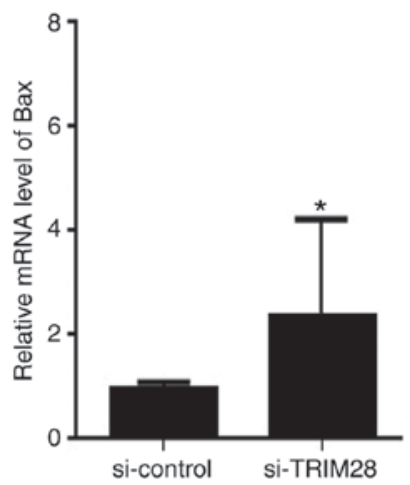

E

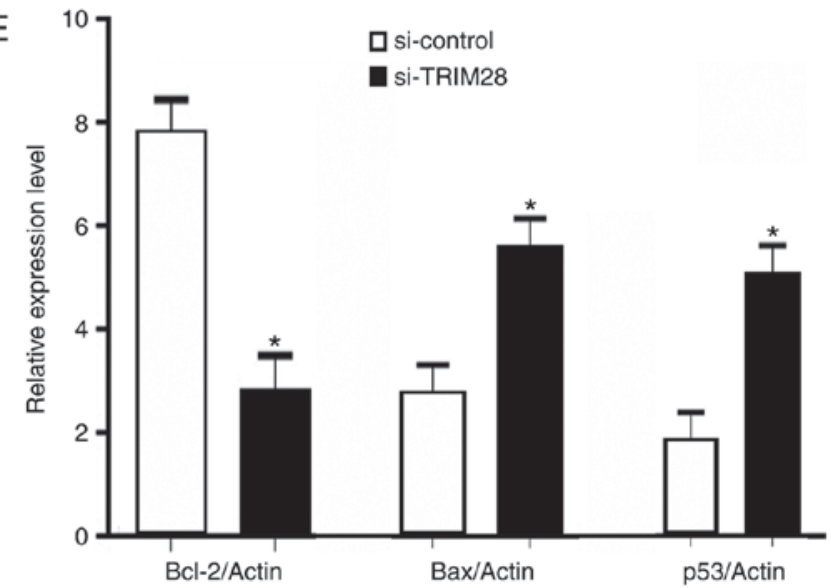

Figure 3. TRIM28 knockdown induced a decrease in Bcl-2 expression and an increase in Bax and p53 expression. (A-C) Comparison of the mRNA expression levels of Bcl-2, Bax and p53 in the TRIM28-siRNA and control-siRNA groups by RT-qPCR. (D). Western blot analysis of the protein expression of Bcl-2, Bax, and 553 in the two groups. (E) Comparison of the protein expression levels of Bcl-2, Bax, and p53 in the two groups. " $\mathrm{P}<0.001$ vs. si-control. Bcl-2, B cell lymphoma-2; Bax, Bcl-2 associated X protein; si, small interfering; RT-qPCR; reverse transcription-quantitative polymerase chain reaction. 
Table I. Patient characteristics and the positive rate of TRIM28 serum autoantibodies in 138 non-small lung cancer patients.

\begin{tabular}{lccc}
\hline & \multicolumn{2}{c}{ NSCLC } & Healthy \\
\cline { 2 - 3 } Characteristics & $\mathrm{n}$ & positive rate (\%) & control (n) \\
\hline Age, years & & & \\
$>60$ & 74 & $9(12.16)$ & 45 \\
$<60$ & 64 & $8(12.50)$ & 35 \\
Sex & & & \\
Male & 88 & $11(12.50)$ & 32 \\
Female & 50 & $6(12.00)$ & 48 \\
Pathology & & & \\
SCC & 51 & $4(9.80)$ & \\
Ade & 58 & $11(18.97)$ & \\
LCLC & 29 & $2(6.90)$ & \\
Clinical stages & & & \\
I and II & 97 & $14(14.43)$ & \\
III and IV & 41 & $3(7.32)$ & \\
Tumor differentiation & & & \\
Good & 36 & $4(11.11)$ & \\
Moderate & 62 & $9(14.52)$ & \\
Poor & 40 & $4(10.00)$ & \\
\hline
\end{tabular}

NSCLC, non-small cell lung cancer; SCC, squamous cell carcinoma, Ade, adenocarcinoma; LCLC, large cell lung cancer.

decreased after the knockdown of TRIM28 in gastric cancer cell lines (15). Here, we also showed that TRIM28 knockdown significantly inhibited NSCLC tumor grow th in nude mice. These results suggest that TRIM28 promoted tumor progression and provided a survival advantage to tumor cells. Therefore, the knockdown of TRIM28 may be a therapeutic target for several tumors. In our study, the H\&E staining of histology sections suggests that TRIM28 knockdown may play a role in antiangiogenesis, which may be a putative mechanism of inhibition of tumor formation. However, further studies are necessary to elucidate the underlying mechanisms.

The present study showed that the knockdown of TRIM28 significantly induced apoptosis compared to the control group $(\mathrm{P}<0.001)$. Moreover, TRIM28 knockdown increased the expression of Bax and p53 and decreased the expression of $\mathrm{Bcl}-2$ at both the gene and protein levels. Wang et al (28) demonstrated that TRIM28 interacted with MDM2, which stimulated the formation of the p53-HDAC1 complex. Furthermore, the knockout of TRIM28 promoted the acetylation and transcriptional activity of $\mathrm{p} 53$, sensitized the response of p53 to DNA damage, and increased apoptosis. A study showed that TRIM28 regulated apoptosis induced by the E2F1 signaling pathway by binding to E2F1 transcription factors in a retinoblastoma protein-independent manner and inhibiting E2F1 activity (29). Another study suggests that TRIM28 plays a role in other signaling pathways associated with apoptosis in tumorigenesis. Kamitani et al (30) found that TRIM28 associated with endogenous STAT1 repressed the interferon-mediated signaling pathway. A siRNA-mediated

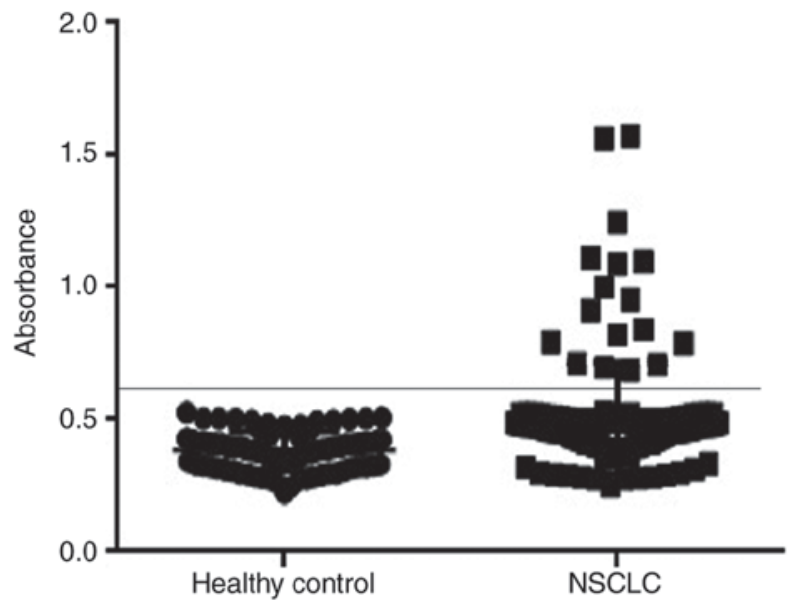

Figure 4. Detection of antibodies to TRIM28 in the sera of NSCLC patients $(n=138)$ and healthy patient $(n=80)$ determined by reverse ELISA. Seropositivity in NSCLC patients was considered the cases in which an absorbance was greater than the mean control absorbance \pm 2 SD. NSCLC, non-small cell lung cancer; ELISA, enzyme-linked immunosorbent assay; $\mathrm{SD}$, standard deviation.

reduction in TRIM28 expression enhanced interferon-induced STAT1-dependent IRF-1 gene expression. Tsuruma et al (31) found that endogenous TRIM28 associated with endogenous STAT3 in vivo. The siRNA-mediated reduction in TRIM28 expression enhanced interleukin-6-induced STAT3-dependent transcription and gene expression and the marked accumulation of STAT3 phosphorylated on Ser727 in the nucleus. Although these studies suggested that TRIM28 may play a role in many signal pathways of apoptosis, the mechanism underlying this role are not clear.

In our study, the subcutaneous xenograft model in nude mice was set up to demonstrate that the silencing of TRIM28 expression by a siRNA lentivirus vector inhibited the growth of NSCLC. If the further roles of TRIM28 are to be explored, influencing metastasis in particular, a clinically relevant orthotopic model of lung cancer put forth by the recent researches (32-34) is needed. Human lung cancer cell lines were genetically transformed to express fluorescent protein. Tumors were grown subcutaneously for each cell line and harvested and minced for surgical orthotopic implantation on the lung of nude mice. Tumor growth and metastasis were measured by fluorescence imaging (34).

Several studies have shown that TRIM28 may be a valuable biomarker for tumor diagnosis and prognosis prediction. Wang et al (35) demonstrated that TRIM28 was overexpressed in human hepatoma cell lines and hepatocellular cancer (HCC) tumor tissues. Moreover, the abnormal expression of TRIM28 was strongly correlated with the clinical stage and tumor size and thus this molecule might be a predictor of poor prognosis in HCC patients. The correlation between TRIM28 expression and clinicopathological features was studied in ovarian cancer (17). The results of multivariate analysis showed that TRIM28 overexpression was an independent predictor of cancer and was correlated with clinical stage, pathological grade, metastases and poor survival. Wang et al (36) used RT-qPCR to detect the expression levels of TRIM28 in peripheral blood karyocytes of gastric cancer patients. The results revealed significant correlations between TRIM28, TNM tumor stages, and distant 
metastases. Using a cohort of colorectal cancer patients and non-cancerous controls, Hector et al (37) demonstrated that autoantibodies to TRIM28 were detectable in the sera of several colorectal cancer patients compared to controls.

Using nested real-time PCR, we measured the expression levels of the TRIM28 gene in the circulating cancer cells of these patients in a previous study (5). Here, autoantibodies to TRIM28 were determined in these patients' sera, representing the measurements at the protein level. The positive detection rates were $30.4 \%(42 / 138)$ for the TRIM28 gene and $12.32 \%$ (17/138) for TRIM28 autoantibodies in 138 NSCLC patients. The expression of the TRIM28 gene was detected in the sera of all 17 patients with TRIM28 antibodies in circulating cancer cells. In turn, the expression of TRIM28 gene and TRIM28 autoantibodies was not detected in the 80 normal controls. The detection rates were $29.9 \%$ (29/97) for TRIM28 gene and $14.43 \%$ (14/97) for TRIM28 autoantibodies in early-stage NSCLC, which suggests that the expression of TRIM28 at both the gene and protein levels may be valuable for early diagnosis. In addition, our results indicate that TRIM28 may be a useful marker for the prediction of metastasis and prognosis. The incidence of recurrence and/or metastasis was significantly higher in the TRIM28 gene positive group (20 of 29, 69.0\%, $\mathrm{P}=0.004$ ) than in the TRIM28 gene negative group (25 of 68, $36.8 \%$ ). Similarly, the rate of recurrence and/or metastasis was higher (12 of $14,85.7 \%)$ in early-stage patients with TRIM28 antibodies (10 patients died in this period) than in patients without TRIM28 antibodies (33 of 83, 39.8\%).

In contrast, our findings demonstrated that the autoantibody-positive rate was only $3.72 \%$ in advanced-stage NSCLC patients vs. $14.43 \%$ in early-stage patients. These results suggest that tumor-associated antigens may activate a complete set of specific and nonspecific defense mechanisms in early-stage patients. In turn, in advanced stages, when the host defense mechanisms against cancer are ineffective, tumor cells evade the immune system (immune incompetent patients) and malignant cells grow and expand in an uncontrolled manner.

In conclusion, our results suggest that the knockdown of TRIM28 expression by lenti-siRNA/TRIM28 may inhibit tumor growth and induce cell apoptosis in vivo. In addition, we demonstrated the high level of specificity of TRIM28 autoantibodies in the sera of NSCLC patients. These results suggest that TRIM28 knockdown may be a valuable approach for the treatment of NSCLC and TRIM28 autoantibodies may act as a blood-based tumor marker to detect early- stage NSCLC.

\section{Acknowledgements}

This study was supported by the National Natural Science Foundation of China (81703001), Hebei Natural Science Foundation(H2015406014),Hebei Province TalentEngineering Training Funded Research Projects (A2016002085), University Emphasis Subject of Hebei Province and Emphasis Subject of Chengde Medical College

\section{References}

1. National Lung Screening Trial Research Team, Aberle DR, Berg CD, Black WC, Church TR, Fagerstrom RM, Galen B, Gareen IF, Gatsonis C, Goldin J, et al: The National Lung Screening Trial: Overview and study design. Radiology 258: 243-253, 2011.
2. Asamura H, Goya T, Koshiishi Y, Sohara Y, Eguchi K, Mori M, Nakanishi Y, Tsuchiya R, Shimokata K, Inoue H, et al: A Japanese Lung Cancer Registry study: Prognosis of 13,010 resected lung cancers. J Thorac Oncol 3: 46-52, 2008.

3. Siegel RL, Miller KD and Jemal A: Cancer statistics, 2015. CA Cancer J Clin 65: 5-29, 2015.

4. Aberle DR, DeMello S, Berg CD, Black WC, Brewer B, Church TR, Clingan KL, Duan F, Fagerstrom RM, Gareen IF, et al: Results of the two incidence screenings in the National Lung Screening Trial. N Engl J Med 369: 920-931, 2013.

5. Liu L, Zhao E, Li C, Huang L, Xiao L, Cheng L, Huang X, Song Y and Xu D: TRIM28, a new molecular marker predicting metastasis and survival in early-stage non-small cell lung cancer. Cancer Epidemiol 37: 71-78, 2013.

6. Messerschmidt DM, de Vries W, Ito M, Solter D, Ferguson-Smith A and Knowles BB: Trim28 is required for epigenetic stability during mouse oocyte to embryo transition. Science 335: 1499-1502, 2012.

7. Barde I, Rauwel B, Marin-Florez RM, Corsinotti A, Laurenti E, Verp S, Offner S, Marquis J, Kapopoulou A, Vanicek J and Trono D: A KRAB/KAP1-miRNA cascade regulates erythropoiesis through stage-specific control of mitophagy. Science 340: 350-353, 2013.

8. Pfeifer GP: Protein phosphatase PP4: Role in dephosphorylation of KAP1 and DNA strand break repair. Cell Cycle 11: 2590-2591, 2012.

9. White DE, Negorev D, Peng H, Ivanov AV, Maul GG and Rauscher FJ III: KAP1, a novel substrate for PIKK family members, colocalizes with numerous damage response factors at DNA lesions. Cancer Res 66: 11594-11599, 2006.

10. Gilmore-Hebert M, Ramabhadran R and Stern DF: Interactions of ErbB4 and Kap1 connect the growth factor and DNA damage response pathways. Mol Cancer Res 8: 1388-1398, 2010.

11. Hu C, Zhang S, Gao X, Gao X, Xu X, Lv Y, Zhang Y, Zhu Z, Zhang $\mathrm{C}, \mathrm{Li} \mathrm{Q}$, et al: Roles of kruppel-associated box (KRAB)associated co-repressor KAP1 ser-473 phosphorylation in DNA damage response. J Biol Chem 287: 18937-18952, 2012.

12. Lin LF, Li CF, Wang WJ, Yang WM, Wang DD, Chang WC, Lee WH and Wang JM: Loss of ZBRK1 contributes to the increase of KAP1 and promotes KAP1-mediated metastasis and invasion in cervical cancer. PLoS One 8: e73033, 2013.

13. Addison JB, Koontz C, Fugett JH, Creighton CJ, Chen D, Farrugia MK, Padon RR, Voronkova MA, McLaughlin SL, Livengood RH, et al: KAP1 promotes proliferation and metastatic progression of breast cancer cells. Cancer Res 75: 344-355, 2015.

14. Fitzgerald S, Sheehan KM, O'Grady A, Kenny D, O'Kennedy R, Kay EW and Kijanka GS: Relationship between epithelial and stromal TRIM28 expression predicts survival in colorectal cancer patients. J Gastroenterol Hepatol 28: 967-974, 2013.

15. Yokoe T, Toiyama Y, Okugawa Y, Tanaka K, Ohi M, Inoue Y, Mohri Y, Miki C and Kusunoki M: KAP1 is associated with peritoneal carcinomatosis in gastric cancer. Ann Surg Oncol 17: 821-828, 2010.

16. Zhang L, Zhu C, Guo Y, Wei F, Lu J, Qin J, Banerjee S, Wang J, Shang H, Verma SC, et al: Inhibition of KAP1 enhances hypoxia-induced Kaposi's sarcoma-associated herpesvirus reactivation through RBP-Jк. J Virol 88: 6873-6884, 2014.

17. Cui Y, Yang S, Fu X, Feng J, Xu S and Ying G: High levels of KAP1 expression are associated with aggressive clinical features in ovarian cancer. Int J Mol Sci 16: 363-377, 2014.

18. Hu M, Fu X, Cui Y, Xu S, Xu Y, Dong Q and Sun L: Expression of KAP1 in epithelial ovarian cancer and its correlation with drug-resistance. Int J Clin Exp Med 8: 17308-17320, 2015.

19. Chen L, Muñoz-Antonia T and Cress WD: Trim 28 contributes to EMT via regulation of E-cadherin and $\mathrm{N}$-cadherin in lung cancer cell lines. PLoS One 9: e101040, 2014.

20. Czerwińska P, Shah PK, Tomczak K, Klimczak M, Mazurek S, Sozańska B, Biecek P, Korski K, Filas V, Mackiewicz A, et al: TRIM28 multi-domain protein regulates cancer stem cell population in breast tumor development. Oncotarget 8: 863-882, 2017.

21. Du ZY, Shi MH, Ji CH and Yu Y: Serum pleiotrophin could be an early indicator for diagnosis and prognosis of non-small cell lung cancer. Asian Pac J Cancer Prev 16: 1421-1425, 2015.

22. Halvorsen AR, Bjaanæs M, LeBlanc M, Holm AM, Bolstad N, Rubio L, Peñalver JC, Cervera J, Mojarrieta JC, López-Guerrero JA, et al: A unique set of 6 circulating microRNAs for early detection of non-small cell lung cancer. Oncotarget 7: 37250-37259, 2016. 
23. Tas F, Bilgin E, Tastekin D, Erturk K and Duranyildiz D: Clinical significance of serum laminin levels in patients with lung cancer. Biomed Rep 4: 485-488, 2016.

24. Sun M, Song J, Zhou Z, Zhu R, Jin H, Ji Y, Lu Q and Ju H: Comparison of serum microRNA21 and tumor markers in diagnosis of early non-small cell lung cancer. Dis Markers 2016: $3823121,2016$.

25. Sreseli RT, Binder H, Kuhn M, Digel W, Veelken H, Sienel W, Passlick B, Schumacher M, Martens UM and Zimmermann S: Identification of a 17-protein signature in the serum of lung cancer patients. Oncol Rep 24: 263-270, 2010.

26. Liu L, Xiao L, Liang X, Chen L, Cheng L, Zhang L, Wu X, Xu Q and Ma C: TRIM28 knock-down increases sensitivity to etoposide by upregulating E2F1 in non-small cell lung cancer. Oncol Rep 37: 3597-3605, 2017.

27. Bojkowska K, Aloisio F, Cassano M, Kapopoulou A, Santoni de Sio F, Zangger N, Offner S, Cartoni C, Thomas C, Quenneville S, et al: Liver-specific ablation of Krüppel-associated box-associated protein 1 in mice leads to male-predominant hepatosteatosis and development of liver adenoma. Hepatology 56: 1279-1290, 2012.

28. Wang C, Ivanov A, Chen L, Fredericks WJ, Seto E, Rauscher FJ III and Chen J: MDM2 interaction with nuclear corepressor KAP1 contributes to p53 inactivation. EMBO J 24: 3279-3290, 2005.

29. Wang C, Rauscher FJ III, Cress WD and Chen J: Regulation of E2F1 function by the nuclear corepressor KAP1. J Biol Chem 282: 29902-29909, 2007.

30. Kamitani S, Ohbayashi N, Ikeda O, Togi S, Muromoto R, Sekine Y, Ohta K, Ishiyama $\mathrm{H}$ and Matsuda T: KAP1 regulates type I interferon/STAT1-mediated IRF-1 gene expression. Biochem Biophys Res Commun 370: 366-370, 2008.

31. Tsuruma R, Ohbayashi N, Kamitani S, Ikeda O, Sato N Muromoto R, Sekine Y, Oritani K and Matsuda T: Physical and functional interactions between STAT3 and KAP1. Oncogene 27: 3054-3059, 2008.
32. Rashidi B, Yang M, Jiang $\mathrm{P}$, Baranov E, An Z, Wang X, Moossa AR and Hoffman RM: A highly metastatic Lewis lung carcinoma orthotopic green fluorescent protein model. Clin Exp Metastasis 18: 57-60, 2000

33. Rashidi B, Moossa AR and Hoffman RM: Specific route mapping visualized with GFP of single-file streaming contralateral and systemic metastasis of Lewis lung carcinoma cells beginning within hours of orthotopic implantation [correction of implantion]. J Cell Biochem 114: 1738-1743, 2013.

34. Yano S, Zhang Y, Miwa S, Kishimoto H, Urata Y, Bouvet M, Kagawa S, Fujiwara T and Hoffman RM: Precise navigation surgery of tumours in the lung in mouse models enabled by in situ fluorescence labelling with a killer-reporter adenovirus. BMJ Open Respir Res 2: e000096, 2015.

35. Wang Y, Jiang J, Li Q, Ma H, Xu Z and Gao Y: KAP1 is overexpressed in hepatocellular carcinoma and its clinical significance. Int J Clin Oncol 21: 927-933, 2016.

36. Wang YY, Li L, Zhao ZS and Wang HJ: Clinical utility of measuring expression levels of KAP1, TIMP1 and STC2 in peripheral blood of patients with gastric cancer. World J Surg Oncol 11: 81, 2013.

37. Hector S, Chen H, Kijanka G, Murray F and Prehn JH: A reverse-ELISA for the detection of TRIM28/KAP1 serum autoantibodies in colorectal cancer patients. Acta Oncol 51: 394-396, 2012.

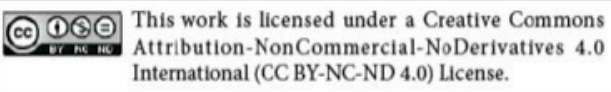

